\title{
Stiffness Imaging with a Continuum Appendage: Real-time Shape and Tip Force Estimation from Base Load Readings
}

\author{
S.M.Hadi Sadati ${ }^{\dagger}$ and Ali Shiva ${ }^{\dagger}$, Nicolas Herzig, Caleb D. Rucker, Helmut Hauser, Ian D. Walker, \\ Christos Bergeles, Kaspar Althoefer, Thrishantha Nanayakkara
}

\begin{abstract}
In this paper, we propose benefiting from load readings at the base of a continuum appendage for real-time forward integration of Cosserat rod model with application in configuration and tip load estimation. The application of this method is successfully tested for stiffness imaging of a soft tissue, using a 3-DOF hydraulically actuated braided continuum appendage. Multiple probing runs with different actuation pressures are used for mapping the tissue surface shape and directional linear stiffness, as well as detecting non-homogeneous regions, e.g. a hard nodule embedded in a soft silicon tissue phantom. Readings from a 6-axis force sensor at the tip is used for comparison and verification. As a result, the tip force is estimated with 0.016-0.037 $\mathrm{N}(7-20 \%)$ mean error in the probing and 0.02-0.1 $N(6-12 \%)$ in the indentation direction, $0.17 \mathrm{~mm}(14 \%)$ mean error is achieved in estimating the surface profile, and 3.4$15[\mathrm{~N} / \mathrm{m}](10-16 \%)$ mean error is observed in evaluating tissue directional stiffness, depending on the appendage actuation. We observed that if the appendage bends against the slider motion (toward the probing direction), it provides better horizontal stiffness estimation and better estimation in the perpendicular direction is achieved when it bends toward the slider motion (against the probing direction). In comparison with a rigid probe, $\approx 10$ times smaller stiffness and $\approx 7$ times larger mean standard deviation values were observed, suggesting the importance of a probe stiffness in estimation the tissue stiffness.
\end{abstract}

Index Terms-Continuum Appendage, Shape Estimation, Force Estimation, Stiffness Imaging, Palpation, Cosserat Rod.

\section{INTRODUCTION}

Recent improvements in soft robotics promise designs for applications that require high dexterity, compliance, and safe

Manuscript received: September, 10, 2019; Revised December 15, 2019 ; Accepted January, 13, 2020.

This paper was recommended for publication by Editor Kyu-Jin Cho upon evaluation of the Associate Editor and Reviewers' comments. This work is Supported by the U.K. EPSRC MOTION [EP/N03211X/2] and RoboPatient [EP/T00603X/1] Grants awarded to T.N., European Union H2020 [637095] awarded to K.A., Leverhulme Trust Project [RPG-2016-345] awarded to H.H., and ERC Starting Grant [714562] awarded to C.B.

S.M.H. Sadati and C. Bergeles are with the Dep. of Imaging and Biomedical Engineering, King's College London, U.K. A. Shiva and T. Nanayakkara are with the Dyson School of Design Engineering, Imperial College London, U.K. A. Shiva is also with the Dep. of Informatics, King's College London, U.K. N. Herzig is with the Department of Automatic Control and Systems Engineering, The University of Sheffield, U.K. C. Rucker is with the University of Tennessee, Knoxville, TN, USA. H. Hauser is with the Department of Engineering Mathematics, University of Bristol, Bristol, U.K. I. D. Walker is with the Dep. of Electrical and Computer Engineering, Clemson University, Clemson, SC, USA. K. Althoefer is with the School of Engineering and Materials Science, Queen Mary University of London, U.K. Correspondence: smh_sadati@kcl.ac.uk. ${ }^{\dagger}$ Equal contributions.

Digital Object Identifier (DOI): see top of this page.

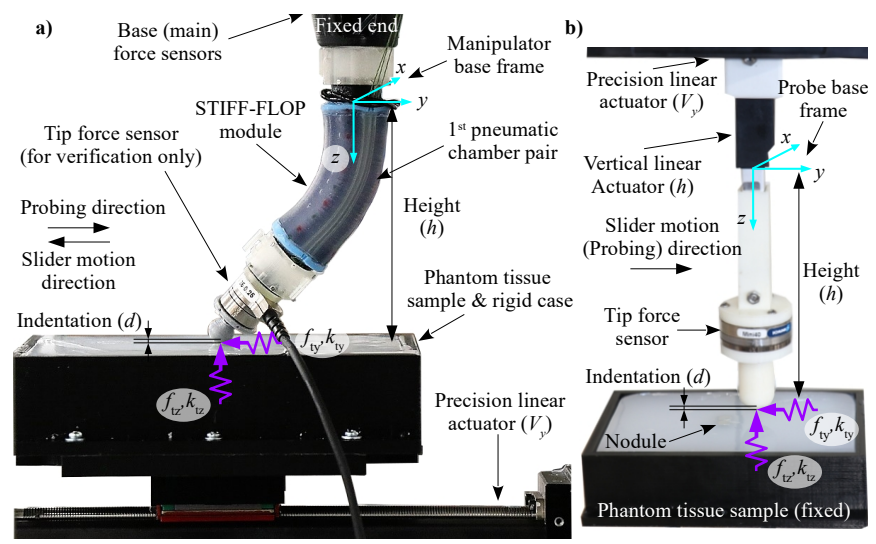

Fig. 1: a) A hydraulically actuated continuum appendage (STIFF-FLOP) for soft tissue probing and stiffness imaging, b) a rigid probe used for experimental comparison.

interaction which the traditional rigid body robots are not suitable for, such as medical and surgical applications, safe manipulation and interaction, and inspection tasks in unstructured environment. However, soft robots dexterity poses challenges with the applications involving real-time modeling and force estimation tasks, such as soft tissue palpation and manipulation. In this study, we propose using a simple yet accurate stiffness estimation method for continuum manipulators that solely relies on readings from a 6-axis force sensor and forward integration of Cosserat rod theory. We showcase this method for probing and stiffness imaging soft tissue organs, e.g. a patient abdomen in a diagnosis scenario, with a hydraulic continuum appendage. To the best of our knowledge, this is the first time that:

- forward integration of Cosserat rod theory based on base force reading is proposed for contact position and force estimation a continuum manipulator,

- the application of a continuum appendage is investigated for stiffness imaging of soft phantom tissue.

Among different robotic solutions for medial applications, the class of continuum manipulators are particularly attractive, exploiting their robust fluidic or tendon actuation mechanisms, high dexterity and intrinsic structural compliance. These features enable safe interaction with soft tissue, high maneuverability and large workspace for physical examination and rehabilitation, reaching complex confined anatomical paths in narrow port Minimally Invasive Surgeries (MIS), and providing intrinsic shape and force sensing through their body or actuation lines [1], [2]. 
Among different designs, braided fluidic continuum modules, such as STIFF-FLOP (STIFFness controllable Flexible and Learn-able manipulator for surgical OPerations) [3], perform better when robust, homogeneous and repeatable deformation and force control are needed, compared to the non-braided versions. However, their real-time and accurate modeling, observation and control in the dynamic environments of medical examination are challenging [4], [5].

Hybrid force and position estimation and control methods have been introduced to address these issues, relying on combining force and position sensor readings to achieve the estimation and control tasks [6], [7]. Bajo et al. recently proposed a hybrid position/force control paradigm for a miniature tendon driven continuum manipulator and showcased it for stiffness imaging of soft tissue. Relying on both whole robot configuration and base force measurements, the authors of [7] compared the force/position data from two palpation rounds to estimate the shape and linear stiffness of an unknown soft environment [7]. Recent research has focused on body or tip (operation point) force estimation to remove the need to attach a force sensor on the manipulator. These methods rely on intrinsic force sensing, through the manipulator actuation tendons [8], [7] and pressure lines [9], and position tracking methods based on tendon length [7], magnetic markers [9], intraoperative methods using fluorescent, ultrasound or visual tracking markers, or more recently, fiber optic and fiber bragg grating sensors [10], [2].

Relying on simplifying assumptions about the manipulator geometry, e.g. the constant curvature [10], the need for expensive and bulky tracking devices with limited range and portability, occlusion problems, MRI interference, and noisy results [2] are the main disadvantages of these shape-based and indirect force estimation methods. More recently, Aloi et al. have proposed an optimization based approach assuming a series solution for point or distributed loads along a planar continuum rod [11]. This method relays on knowing the rod configuration and does not necessarily result in a unique solution for the forces.

To simplify the shape estimation of a continuum appendage, the idea of transforming the Boundary Value Problem (BVP) model of an elastic beam to an Ordinary Differential Equation (ODE) by knowing the loading condition at a fixed-end has been utilized by Barbič for soft material graphical visualizing [12]. From a robotic point of view, Bretl and Mccarthy have showed that the reaction forces at one end of an elastic rod are global coordinate charts for estimating the rod quasistatic configuration [13]. Experimental investigation of this concept is carried out for modeling thin solids, e.g. flexible ribbons [14], [15]. Recently, Takano et al. employed this idea for shape estimation of continuum rods with moving ends achieving simple forward-integration, real-time performance, small error (3-15\%) and occlusion free tracking, but limited accuracy due to discrete implementation of the Kirchhoff Elastic Rod method [15]. Rucker et al. have briefly investigated this idea as a part of their actuation- and deflection-based force sensing method for parallel continuum robots [16]. However, geometric constraint (actuation port overlapped with base force sensor placement) and large sensitivity of the method to noise in dynamic scenarios prevented them from effectively implementing the idea. To the best of our knowledge, this is for the first time that the concept of using a Cosserat rod model of a continuum appendage is used to estimate the tip force and position based on the base force readings, in a tissue stiffness imaging application using a pneumatically actuated braided continuum manipulator.

Various probing devices, including tactile sensors, have been developed to minimize medical intervention damage for graspers, forceps, cutters, catheter tips, or to detect presence of abnormalities by evaluating tissue mechanical properties and providing tactile feedback [17]. While robust solutions for static single point stiffness measurements already exist, realtime accurate and stable stiffness mapping of a tissue surface is still challenging. The tissue shape and stiffness estimation are possible by keeping a zero-force contact with the tissue surface, requiring a force/position controller, [18], [19], [7] and then comparing the indentation due to different applied force values from multiple palpation runs [20].

In this paper, we propose a real-time shape and tip force estimation method based on forward numerical integration of Cosserat rod method that is made possible using 6-axis load readings from a force sensor fixed at the appendage base (section II-A \& II-B). As a result, no position tracking sensor is needed and the stiffness imaging algorithm is simple and efficient enough to be implemented in an affordable easyto-use micro-controller. The use of a hydraulically actuated STIFF-FLOP module, which is a 3-DOF braided continuum appendage, is proposed for probing and stiffness imaging of a soft silicon tissue phantom, as in Fig. 1-a, with the aim of detecting anomalies in the form of a hard nodules in the soft tissue phantom (section II-C)

Hyperelasticity and braiding effects are taken into consideration in an intermediate numerical step as in [21], [22]. The results from multiple probing runs with different actuation pressures provide sufficient information to construct the surface profile and the linear stiffness map of the phantom tissue with good accuracy compared to experimental results, showing the location of the anomalies in the tissue. Further analysis is provided based on comparison with experimental results from a rigid probe as in Fig. 1-b Suggestions for future research are provided based on a discussion on the experimental and simulation results (section III). Conclusions are presented in section IV.

The main contributions of this study can be summarized as: (i) proposing real-time shape and tip force estimation via sensing the appendage base loads and hence enabling forward integration of a Cosserat rod model static case, (ii) investigating the accuracy and real-time computational performance of the continuum appendage mechanics using rotation matrices, (iii) using a continuum actuator appendage for soft tissue palpation and experimental comparison with a rigid probe, and (iv) suggestions for probing strategies with a continuum appendage. There is no need for a tip force sensor in the proposed method, although one is used in this research for experimental verification. 


\section{MAterials AND Methods}

\section{A. Continuum Appendage Static Model}

Variable Curvature (VC) kinematics, based on rotation matrices and Cosserat rod theory are used to model the static mechanics of the appendage [1], [23]. The local physical curvilinear coordinates are $\left[\hat{d}_{1}, \hat{d}_{2}, \hat{d}_{3}\right]$, where $\hat{d}_{3}$ is tangent to the backbone, $\hat{d}_{2}$ is along the first pressure chamber pair, and $\left[\hat{d}_{1}, \hat{d}_{2}, \hat{d}_{3}\right]_{(s=0)}=[\hat{i}, \hat{j}, \hat{k}]$ at the appendage base (Fig. 2). Note the orientation of the base frame in Fig 1 . The backbone curve spatial configuration $(\rho)$ and $3 \times 3$ rotation matrix $(R)$, expressed in inertial Cartesian coordinates $([\hat{i}, \hat{j}, \hat{k}])$, are derived based on VC as [1], [24]

$$
\rho_{, s}=R\left(v+[0,0,1]^{\top}\right), R_{, s}=R[u]_{\times},
$$

where $R$ is the $3 \times 3$ rotation matrix, $v$ is the local strain vector, $u$ is the vector of curve local curvatures and torsion, $s$ is the variable along the backbone with $s_{\text {tip }}=l, l$ is the manipulator length, $y_{, x}=\partial y / \partial x$, and []$_{\times}$is the skew-symmetric matrix denoting a standard mapping from $\mathbb{R}^{3}$ to $\mathrm{SO}(3)$ [1]. The curvilinear and Cartesian coordinates are aligned at the manipulator base $(s=0)$, where $\left[\hat{d}_{1}, \hat{d}_{2}, \hat{d}_{3}\right]_{(s=0)}=[\hat{i}, \hat{j}, \hat{k}]$, $\rho_{0}=0$ and $q=[0,0,0]$.

The Cosserat rod theory exploits the conservation laws to balance the total internal loads carried by the rod material $(n, m)$ and the distributed external and body loads $\left(f, \tau_{\sigma}\right)$, e.g. due to body weight $\left(f_{\mathrm{g}}\right)$. Here, $m, n_{(s)}$ are equal to the sum of the individual contributions of the actuation chambers $\left(f, \tau_{\mathrm{p}}\right)$ and the projected load on the rod cross-section due to external loads $\left(f, \tau_{\mathrm{m}}\right)$ as $n=f_{\mathrm{p}}+f_{\mathrm{m}}$ and $m=\tau_{\mathrm{p}}+\tau_{\mathrm{m}}$. The resulting load from concentrated external loads $\left(f_{1}, \tau_{1}\right)$ act as boundary conditions (e.g. of type shear load for an Euler-Bernoulli beam), expressed in the local frame, along the manipulator [25]. If the loads due to actuation pressure $\left(f_{\mathrm{p}}, \tau_{\mathrm{p}}\right)$ are considered as concentrated external loads at the manipulator tip and handled as boundary conditions, similar to the case of external $f, \tau_{1}$ [24], complicated terms related to $f_{\mathrm{p}} \hat{d}_{3, s}$ and $\tau_{\mathrm{p}}$ vectors appear in the derivations to compensate for the accumulation of pressure loads as we integrate along the manipulator backbone. Instead, we sum up their contributions to the internal load carried by the rod material $(n, m)$, where they act in parallel to the internal loads $\left(f, \tau_{\mathrm{m}}\right)$ to deform the manipulator. This results in the same set of equations as in [24] but with a clearer and more physically relevant presentation which is less computationally expensive to integrate. For $f, \tau_{\mathrm{m}}$ in the static case, using the Cosserat rod method [1], we have

$$
f_{\mathrm{m}, s}+f_{\sigma}=\mathbf{0}, \tau_{\mathrm{m}, s}+\rho_{, s} \times f_{\mathrm{m}}+\tau_{\sigma}=\mathbf{0},
$$

where $f_{\sigma}=\left(\sigma_{\mathrm{m}} a_{\mathrm{m}}+6 \sigma_{\mathrm{p}} a_{\mathrm{p}}\right) g, \sigma$ are the material and water density, $a_{\mathrm{m}}=\pi\left(r_{\mathrm{m}_{2}}^{2}-r_{\mathrm{m}_{1}}^{2}-6 r_{\mathrm{p}_{1}}^{2}\right)$ and $a_{\mathrm{p}}=\pi r_{\mathrm{p}_{1}}^{2}$ are the manipulator and hydraulic chamber cross-section area respectively, $g=\left[\begin{array}{lll}0 & 0 & -9.81\end{array}\right]\left[\mathrm{m} / \mathrm{s}^{2}\right]$ is the gravity vector, $r_{\mathrm{m}_{1}}$ and $r_{\mathrm{m}_{2}}$ are the module cross-section inner and outer radii. As a standard approach in implementing Cosserat rod method, Hooke's law of linear stress-strain relation is used as the system constitutional law $\left(n=K_{v} \cdot v, m=K_{u} \cdot u\right)$. Transforming all the vectors in the local frame, we have

$$
v=K_{v}^{-1}\left(R^{\top} f_{\mathrm{m}}+f_{\mathrm{p}}\right), u=K_{u}^{-1}\left(R^{\top} \tau_{\mathrm{m}}+\tau_{\mathrm{p}}\right) .
$$

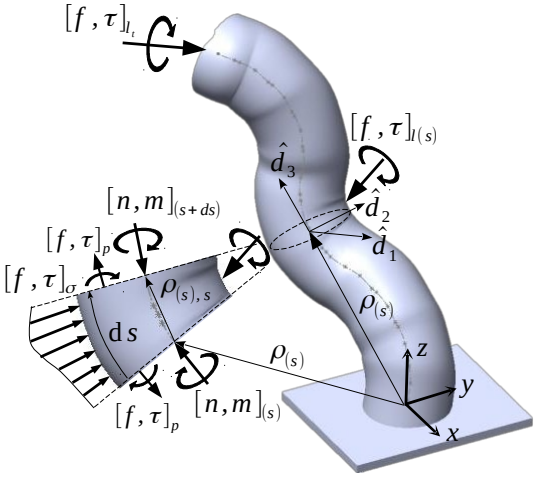

Fig. 2: Variable curvature kinematics and the Cosserat rod method free body diagram for one differential element along the continuum backbone. Subscripts $1, \mathrm{p}$ and $\sigma$ are for the external point loads, loads due to internal pressure, and distributed body loads, e.g. due to gravity, respectively.

where hydraulic chambers are actuated in pairs $\left(p_{2 i-1}=p_{2 i}\right.$ for $i=1 \ldots 3), f_{\mathrm{p}}=\Sigma_{i=1}^{6} p_{i} a_{\mathrm{p}}$ and $\tau_{\mathrm{p}}=\Sigma_{i=1}^{6} p_{i} a_{\mathrm{p}} r_{\mathrm{O}_{i}} \times$ $[0,0,1], \quad r_{\mathrm{p}_{1}}$ and $r_{\mathrm{p}_{2}}$ are the hydraulic chamber inner and outer radius, $K_{v}=\operatorname{diag}\left(a_{\mathrm{m}}[G, G, E]\right)$ and $K_{u}=$ $\operatorname{diag}([E, E, G]) \cdot \operatorname{diag}(J)$ are diagonal stiffness matrices, associated with strains and curvatures/torsion respectively, in the $\hat{d}_{i}$ frame, $E$ and $G \approx E / 3$ are the material elasticity and shear modulus, $J=\pi / 4\left(r_{\mathrm{c}_{2}}^{4}-r_{\mathrm{c}_{1}}^{4}-6 r_{\mathrm{p}}^{4}\right) \cdot[1,1,2]-a_{\mathrm{p}} \operatorname{diag}\left(r_{\mathrm{O}} \cdot r_{\mathrm{O}}^{\top}\right)$ is a $1 \times 3$ vector consisting of the cross-section second moments of areas, $r_{\mathrm{O}}$ is a matrix of which rows are position vectors of the chambers in the manipulator cross-section plane

$$
\begin{aligned}
r_{\mathrm{O}_{i}} & =r_{\mathrm{o}} \cdot\left[\cos \left(\psi_{\mathrm{o}_{i}}\right), \sin \left(\psi_{\mathrm{o}_{i}}\right), 0\right], i \in 1 \ldots 6 \\
\psi_{\mathrm{o}_{2 j-1}} & =\pi / 2-2(j-1) \pi / 3-\phi_{\mathrm{o}}, \\
\psi_{\mathrm{o}_{2 j}} & =\pi / 2-2(j-1) \pi / 3+\phi_{\mathrm{o}}, j \in 1 \ldots 3,
\end{aligned}
$$

and $r_{\mathrm{o}}$ is the radial offset of the chambers from the center. $m, n$ can be used for stress analysis.

Finally, an intermediate step is applied in the numerical integration to account for the braid constraint as $r_{\mathrm{p}}^{*}=$ $r_{\mathrm{p}} \sqrt{1-\lambda^{2} \cos (\gamma)^{2}} / \sin (\gamma)$ [4], and the material hyper-elastic deformation as $r^{*}=r / \sqrt{\lambda}$ and $E^{*}\left|G^{*}=E\right| G / \lambda$ [21], [22], except for $r_{\mathrm{p}}$, which follows the braid constraint. Here, $\lambda=1+v_{2}$ is the local axial stretch along the manipulator backbone. We set $a_{\mathrm{p}}$ and $[f, \tau]_{\mathrm{p}}$ to zero in the equations above when $s<l_{\mathrm{c}}$ and $s>l_{\mathrm{m}}-l_{\mathrm{c}}$ to account for the silicon caps.

Eq. (1), (2), and (3) form a system of differential equations with states $[\rho, R, n, m]$. Benefiting from the load readings from 6-axis force sensor at the manipulator base $\left([f, \tau]_{(s=0)}=\right.$ $\left.[n, m]_{(s=0)}\right)$, the system is reduced to an ODE to be solved with forward numerical integration on $s \in[0, l]$ for the manipulator configuration $(\rho, R)$ and tip load $\left([f, \tau]_{1}=[n, m]_{(s=l)}\right)$. In the case that the tip loads are known, a more computationally demanding and harder to solve BVP is formed with convergence and accuracy issues. Note that reduced-order methods have been introduced recently to solve these systems in [26], [21]. The formed ODE is solved for each time sample, which is equal to the force sensor data acquisition sampling time (0.02 [s]), using Matlab software's "ode113" function, featuring adaptive integration time step and error evaluation. Simulation results for the tip position are used to estimate the 


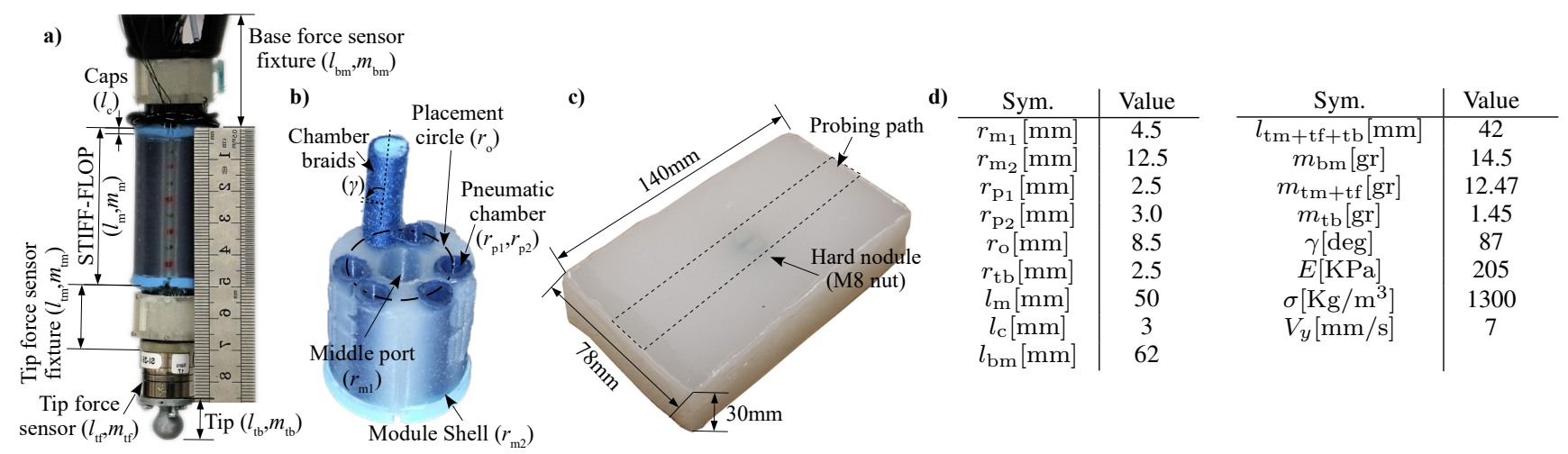

Fig. 3: a) Schematic of the probing appendage consisting of a STIFF-FLOP actuator module, tip and base force sensors, and mountings. b) Internal structure of a STIFF-FLOP module. c) A soft silicon phantom tissue with an embedded M8 nut to mimic a hard nodule in a soft tissue. d) The continuum appendage setup and experiment parameters. indentation of the contact point with the tissue to estimate the tissue profile and stiffness. The simulation results for the tip orientation are used to transform the tip force sensor readings to the base reference frame as the ground truth to be compared with the simulation results based on the base force sensor readings.

\section{B. Appendage Shape and Tissue Stiffness Estimation}

The phantom tissue is moving w.r.t. the appendage in our experiments to avoid complexities due to motion and force control of the appendage. The appendage is fixed with an offset $h$ from the tissue surface. The tip contact position with the tissue w.r.t. the phantom edge in each time step $t$ is $\rho_{\mathrm{t}}=$ $\left[0,-V_{y} t, h\right]-\rho_{(s=l)}$.

Two methods can be used to estimate the tissue stiffness. Assuming the tissue with an ideal flat surface, the tissue stiffness $(k)$ is found for each trial as $k_{y \mid z}=f_{\mathrm{t} y \mid z} /\left(\rho_{\mathrm{t} z}-h\right)$. Alternativaly, the tissue linear stiffness can be estimated by comparing the appendage tip position and forces of different experimental trials with different tissue indentations $(d)$ due to different actuation pressures as $k_{y \mid z}^{*}=\Delta f_{\mathrm{t} y \mid z} / \Delta \rho_{\mathrm{t} z}$ [7], where $k^{*}$ is the tissue linear stiffness w.r.t. $d . k_{y}$ is the apparent stiffness in the probing direction which is related to the surface friction $\left(\mu_{\mathrm{t}}\right)$ and the tissue real stiffness along probing direction $\left(k_{y_{\text {real }}}\right)$ as $k_{y}=k_{z} \mu_{\mathrm{t}}+k_{y_{\text {real }}}$. We continue our study based on $k_{y}$. Using the second approach, we use $f_{\mathrm{t} z}$ and the estimated $k_{z}^{*}$ to estimate the free surface as $z_{\mathrm{t}}=\rho_{\mathrm{t} z}-f_{\mathrm{t} z} / k_{z}^{*}$. This simple procedure does not require a complex force/position control design as in [7] where estimating the tissue surface profile is achieved by zero force probing of the surface. We hypothesize that following our suggested approach, the surface estimation will be less sensitive to the tissue movement in real medical applications (which makes zero force probing a challenging task) and the estimation can be done online while the tip is performing any other task, e.g. if a surgical tool is attached to the tip.

\section{Experimental Setup and Procedure}

A STIFF-FLOP module [3] herein is used as a hydraulically-actuated continuum appendage for soft tissue probing (Fig. 3-a), because of its high repeatability and negligible performance change due to aging and fatigue during the experimental trials. The manipulator is made of silicon elastomer (Ecoflex 0050, Smooth-On Inc.) Independent actuation of three braided hydraulic chamber pairs ( 6 chambers in total) is via separate plungers $(10 \mathrm{~mL}$ Terumo Syringe) moved by 17HS5001-100D8 non-captive stepper motors, provide 3-DOF (one axial elongation and two side-bending) of the appendage tip (Fig. 3-b). Control of the stepper motors are facilitated via a C\# program through a data acquisition board (DAQ) board (National Instruments Inc. NI-DAQmx USB-6411). Hydraulic pressure for each chamber pair is recorded via 3 separate pressure transmitters (WIKA model A-10). The first chamber pair is placed along the manipulator $+y$-axis (Fig. 1) with 120 [deg] offset from the other pairs. Chambers in a pair have $\approx 40$ [deg] offset with each other $\left(\phi_{\mathrm{o}} \approx 20\right.$ [deg] w.r.t. their symmetry line).

An ATI Mini40 F/T sensor is mounted at the appendage base as the single necessary sensor for the probing task. An ATI Nano17 F/T force sensor is connected at the manipulator tip to provide ground truth for validation of our method. A 3D printed spherical tip is mounted at the appendage tip to provide smooth sliding on the tissue sample.

The cubic soft silicon tissue phantom $(140 \times 80 \times 30[\mathrm{~mm}])$ is made from soft silicon (Ecoflex 0010) and confined in a 3D printed rigid container (Fig. 3.c). A M8 nut (a hexagonal with $\approx 14.4[\mathrm{~mm}]$ circumferential circle diameter and $\approx 7.2$ [mm] thickness) is embedded vertically at the middle of the phantom and in $2[\mathrm{~mm}]$ depth, mimicking a hard nodule in a soft tissue. The measured and identified structural parameters of the experimental setup are presented in Fig. 3. The phantom is fixed on a HIWIN KKA40 high precision linear actuator which is controlled with Arduino through a stepper motor.

The appendage is fixed at the target configuration, and the phantom is moved opposite the $y$-axis $(-y)$, as in Fig. 1, with constant velocity $\left(V_{y} \approx 7[\mathrm{~mm} / \mathrm{s}]\right)$ using the linear actuator while in contact with the appendage tip. This configuration is used in the current study for simplicity; however, the results are similar to the case of a fixed phantom and a horizontally moving appendage. An alternative approach is palpating by moving the appendage itself using its hydraulic actuators which we postpone to a future study due to complications that arise for the appendage tip position and force control which are beyond our goals in this study. The phantom tissue is placed $h=89[\mathrm{~mm}]$ away from the appendage base $(2[\mathrm{~mm}]$ less 
than the appendage overall height).

Alternatively, a rigid probe is used for comparison purposes. A rigid probe consisting of 3D-printed mountings for an ATI 6-axis force sensor at the tip of the probe and a nob-shape tip is mounted on a vertical L16-P micro linear actuator (6 [mm] stroke) and a horizontal precision linear actuator. The probe height and hence the probe indentation in the tissue are adjusted by the vertical linear actuator. The phantom tissue is kept fixed, while the horizontal linear actuator slides the probe on the tissue surface.

Four actuation scenarios are tested, each three times, with different input pressure values and probe type (Table I).

I. $p_{\mathrm{I}}=[0.25,0.42,0.5]$ where the appendage bends against the slider motion (toward the probing direction),

II. $p_{\text {II }}=[0.41,0,0]$ where it bends toward the slider motion (against the probing direction),

III. $p_{\text {III }}=[0,0,0][$ atm $]$ where it is neutral (not actuated).

IV. probing experiments with a rigid probe.

The appendage in scenario III bends slightly toward the slider motion passively. The mean (M) and Mean Standard Deviation (MSTD) values for the estimated tip force, based on simulations using the base force sensor readings, are compared with the actual measurements from the tip force sensor to evaluate absolute and percentage error (Err,\%) for each actuation scenario. Note that the sensors' measurements are not equal despite the quasi-static assumption. The tip force sensor readings are measured w.r.t. the appendage tip local frame, since the force sensor is fixed to the tip, and we rely on our simulation results to estimate the tip orientation and its contacting point with and indentation in the phantom tissue. In addition, the mass for appendage body, filled hydraulic chambers, and all rigid connections should be taken into account. Result pairs from different actuation scenarios are compared to estimate the tissue stiffness and surface profile, and the mean value of the results based on the base and tip load readings are compared.

\section{RESULTS AND DISCUSSION}

The results from 9 probing runs with 3 different pressure combinations are used to test the accuracy and computational performance of our method. The readings are carried out with $0.02 \mathrm{~s}$ sampling time and then filtered based on a moving average method, using the Matlab "smooth" function, with a window size of 100 samples. The data points for similar locations on the phantom tissue $\left(y_{\mathrm{t}}\right)$ are extracted by linear interpolating, using the Matlab "interp1" function. Fig. 4 shows raw data from a probing run with initial pressures $p_{\text {III }}=[0,0,0]$ [atm], changing to $p_{\mathrm{III}}^{*}=[-0.1,0,0.05]$ [atm] upon tissue contact. The pressures can become negative due to induced suction in the chambers as a result of passive appendage deformation under its weight or other chamber elongation. The change in the pressure readings is used to detect the contact start time and transition period. The stabilized pressures after tissue contact for the other two sets of actuation scenarios are $p_{\mathrm{I}}^{*}=[0.1,0.5,0.7]$ and $p_{\mathrm{II}}^{*}=[0.3,0,0] .[f, \tau]_{\mathrm{b}}$ are used as $[n, m]_{(s=0)}$ and the estimated tip forces are compared with the actual tip force sensor readings. Our method provides real-time
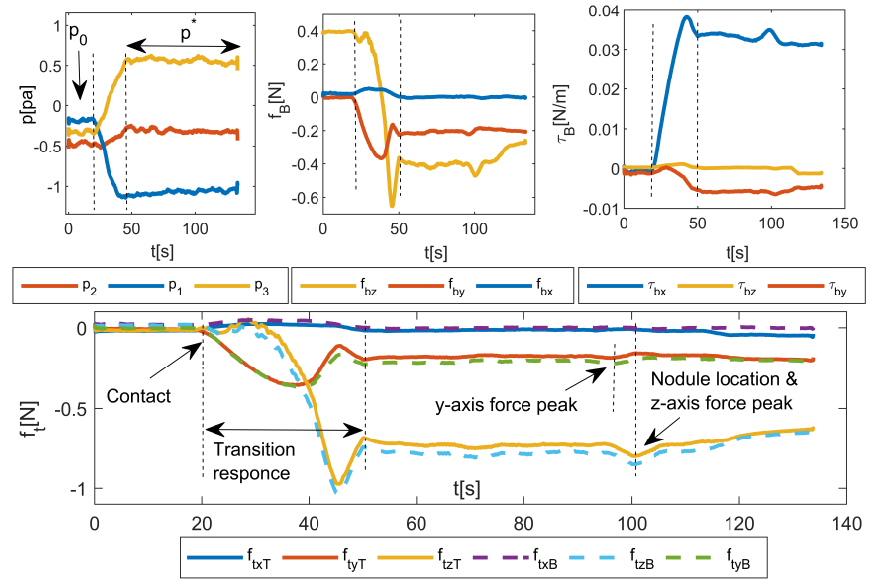

Fig. 4: Sample raw data from a single probing run with $p_{\text {III }}=[0,0,0][\mathrm{atm}]$. The negative pressures $\left(p^{*}\right)$ are due to suction under appendage weight and phantom sliding force. The appendage initial contact is detected by monitoring the pressure reading sudden changes and the transient behavior afterwards. The nodule location (stiff region) is detected upon an increase in the force sensor $z$-axis readings.

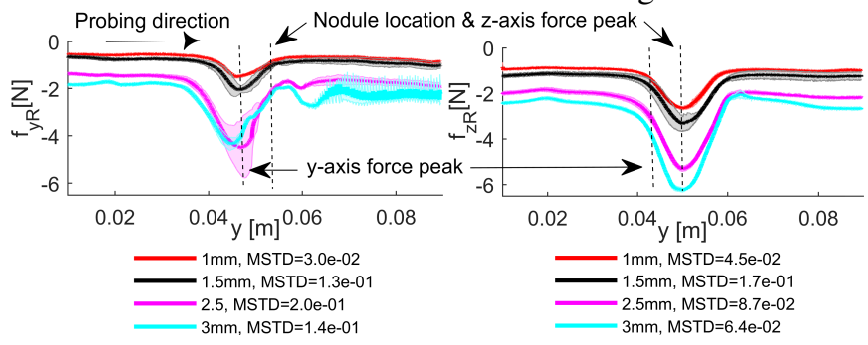

Fig. 5: Tip force $f_{\mathrm{tR}}$ in experiments with a rigid probe with different indentation values.

performance with execution time $364 \mathrm{~ms} / \mathrm{s}$ ([ms] of simulation time per [s] of experiment) running on an Ubuntu 18.04.1 LTS operating system with Intel ${ }^{\circledR}$ Cor ${ }^{\text {TM }}$ M-5Y10c CPU (0.8-2.0 $\mathrm{GHz} \times 4$ ) and $8 \mathrm{~Gb}$ memory). A peak force value is observed at the appendage tip $z$-axis direction almost on top of the stiff region, e.g. hard nodule location, while the $y$-axis (probing) direction peak value occurred just before this point. Subscript $\mathrm{B}$ and $\mathrm{T}$ are used for values referring to the base and tip force sensor measurements respectively.

Fig. 5 shows the experimental results with a rigid probe for four different indentation values. A similar trend with the case of a continuum appendage probe is observed in the peak force values but with $\approx 10$ times larger mean value and variation in the stiff region. Subscript $\mathrm{R}$ is used for values referring to the rigid probe measurements.

Table I presents the MSTD values for all experimental data points, simulation and estimation results of the four probing runs in each of the actuation scenarios. MSTD values are less than $0.02 \mathrm{~N}$ and $0.05 \mathrm{~N}$ for both base and tip force readings in the $y$-axis (probing) and $z$-axis directions showing good repeatability of the experiment results. The same is observed for the moment around the $x$-axis with MSTD less than 1 $[\mathrm{mNm}]$. Scenario I (appendage bends against slider motion) poses the smallest variability of the base force readings in the $y$-axis direction, showing the best accuracy in measuring $f_{\mathrm{t} y}$, and the highest in the $z$-axis direction, showing small 
accuracy in measuring $f_{\mathrm{t} z}$. As a result, the estimated stiffness in the probing direction $\left(k_{y}\right)$ shows the smallest error (2.9 $[\mathrm{N} / \mathrm{m}], 7.1 \%)$ for scenario I when comparing estimations based on the base $\left(k_{y \mathrm{~B}}\right)$ and tip $\left(k_{y \mathrm{~T}}\right)$ force sensor readings. Error analysis results are presented in brackets in the table. Scenario II (appendage bends toward slider motion) shows the smallest variability of this reading in the $z$-axis direction, better for measuring $f_{\mathrm{t} z}$, and scenario III (inactive appendage) shows the highest variability in the $y$-axis direction, not desirable for force measurements in this direction. The estimated stiffness in the indentation direction $\left(k_{z \mathrm{~B}}\right)$ shows the smallest error (2.9 $[\mathrm{N} / \mathrm{m}], 7.1 \%$ ) for scenario II. The lowest variability of base moment readings around $x$-axis occurred in scenario I. The tip force sensor readings show small MSTD values too (less than $0.02 \mathrm{~N}$ ) but they are not considered in our argument about the advantages of each probing configuration, since their readings are in the tip local frame.

Measurements with a rigid probe show larger MSTD for $f_{\mathrm{t}}$ and $k_{y}$ showing the filtering role of the appendage compliant body. However, smaller MSTD is observed for $k_{z}$ suggesting the importance of the probe higher stiffness in achieving higher stiffness estimation reliability. $f_{\mathrm{t} x x}$, and $\tau_{\mathrm{t} y \mid z}$ remain small since our experiments are carried out in planar cases.

The simulation results for the tip forces are reasonably accurate in comparison to the actual readings from the tip force sensor (less than $0.04 \mathrm{~N}$ and $0.9 \mathrm{~N}$ absolute error for $f_{\mathrm{t} y}$ and $f_{\mathrm{t} z}$ respectively). The accuracy of the estimation method is higher for $f_{y}$ in scenario I $(6.7 \%)$ and for $f_{\mathrm{t} z}$ in scenarios II \& III (6.2 \& 6.4\%), supporting our conclusion about the importance of bending direction on measurement axes. Fig. 6 shows a comparison between the tip force value from the tip force sensor readings $\left(f_{\mathrm{tT}}\right)$ and the tip force estimated value based on the base force sensor readings and the proposed theory $\left(f_{\mathrm{tB}}\right)$, and their error analysis for $P_{\mathrm{III}}$. The estimated tissue stiffness $(k)$ based on the base $\left(k_{\mathrm{B}}\right)$ and tip $\left(k_{t}\right)$ force sensors and comparison of the estimated values $\left(k_{\mathrm{Err}}\right)$ are presented in Fig. 7. The appendage shape and tip position are estimated based on the base sensor force readings (values with subscript B) that show higher apparent indentation, w.r.t. the mean height $h=89 \mathrm{~mm}$, in scenario I (bending opposing the probing direction), and smaller values in scenario II (bending toward the slider motion) The plots are trimmed to remove the contact and transient phases. A slightly higher variation in the results are observed near the stiff region of the tissue (on top of the nodule), probably due to a higher force absolute value at the point.

The tissue stiffness $\left(k \& k^{*}\right)$ is calculated, once based on the estimated tip force from the simulations, and once using tip force sensor readings, and compared with each other. In both cases, the estimated tip position from simulations is used. Fig 8-a shows $k_{z \mathrm{~B}}$ estimated values (assuming ideally flat surface), showing high pick values for scenario II (appendage bent toward the probing direction). The same is observed for $k_{z \mathrm{~T}}$, suggesting less accurate tip position estimation in this case. The tissue mean stiffness ( $k$ in Fig $8-\mathrm{b}$ ) is calculated based on results from scenario I \& III.

Alternatively, the tissue exact surface profile $\left(z_{\mathrm{t}}\right)$ and stiffness $\left(k^{*}\right)$ can be calculated by comparing results from two
TABLE I: Mean Standard Deviation (MSTD), and simulation mean error value (Err) and percentage (\%) for the four tested actuation scenarios. The slider moves from right to left.

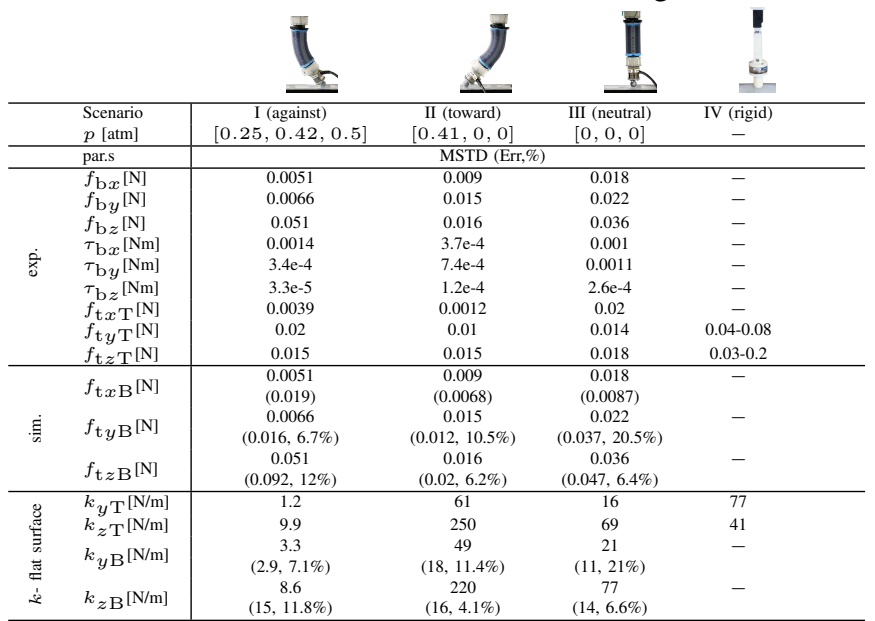

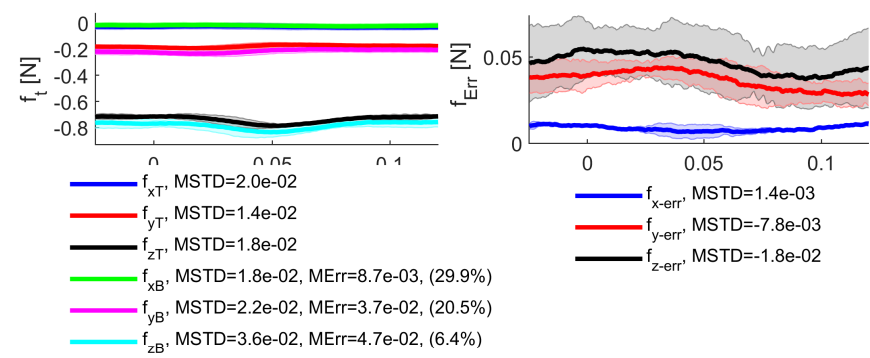

Fig. 6: left) Tip force experimental and estimated values. right) Tip force estimation error analysis.

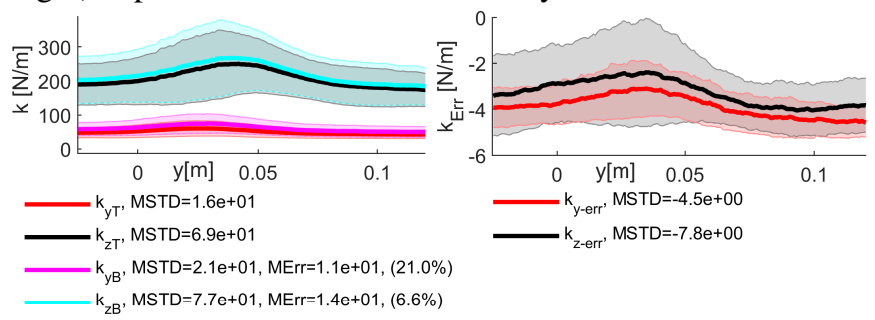

Fig. 7: left) Estimated tip stiffness based on the tip force readings $(\mathrm{T})$ and tip force estimation (B). The appendage shape estimation is used in the both cases. right) Tip stiffness estimation error analysis.

actuation scenarios. The results for three pairs (I-II, I-III, and II-III) are analyzed and the mean values and error analysis are reported in (Fig 8-c \& d). The large difference in the apparent indentation between the actuation scenarios results in high variation in the estimated tissue stiffness and surface profile around the stiff region. The stiffness mean and MSTD values are smaller in the probing direction ( $y$-axis) compared to the indentation direction ( $z$-axis), with overall less variability (better accuracy) around the stiff region. The mean and MSTD values of the calculated values for the tissue surface profile, estimated based on the base force sensor readings $\left(z_{\mathrm{B}}\right)$ or from the direct measurements by the tip force sensor $\left(z_{t \mathrm{~T}}\right)$, are very similar with $\approx 0.17 \mathrm{~mm}(14.2 \%)$ mean error and $<1.8 \mathrm{~mm}$ MSTD value. However, the MSTD values are higher around the stiff region. This results in less accurate surface profile estimation around the stiff region but provides information that eases the nodule detection [27], [19]. The 

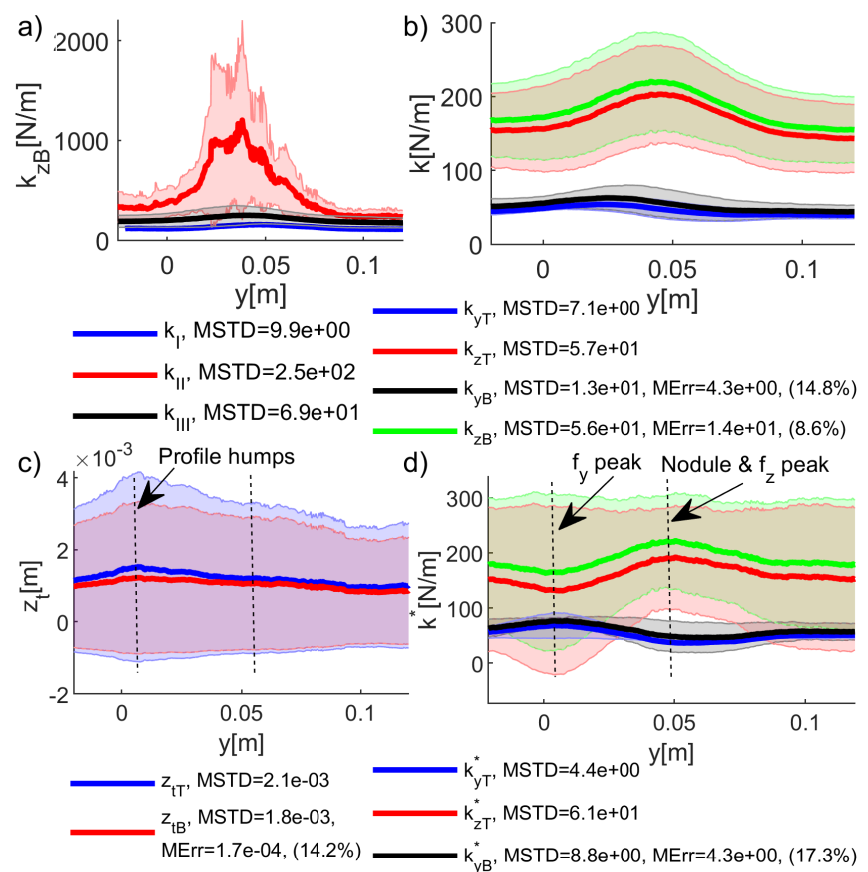

d)
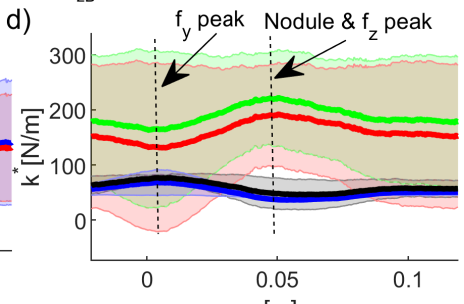

$\mathrm{k}_{\mathrm{yT}}^{*}, \mathrm{MSTD}=4.4 \mathrm{e}+00 \mathrm{y}[\mathrm{m}]$

$\mathrm{k}_{\mathrm{zT}}^{*}, \mathrm{MSTD}=6.1 \mathrm{e}+01$

$\mathrm{k}_{\mathrm{yB}}^{*}, \mathrm{MSTD}=8.8 \mathrm{e}+00, \mathrm{MErr}=4.3 \mathrm{e}+00,(17.3 \%)$

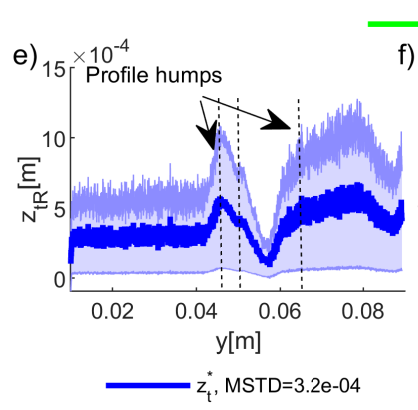

f)

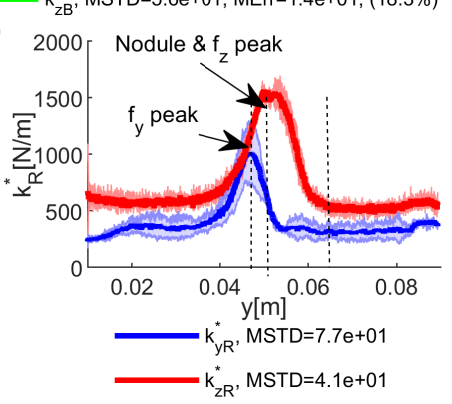

Fig. 8: Error analysis for the tip indentation in the phantom tissue, tissue surface profile and directional stiffness values based on the estimated values from the simulations and the readings from the tip force sensor.

estimated surface profile reveals irregularities in the tissue phantom surface which presents a small hump $(\approx 0.4 \mathrm{~mm})$ just before the embedded nodule, probably due to uneven curing of the phantom tissue surface. The estimated stiffness values from both methods are very similar. While $k$ shows less variability, we needed to identify and filter inaccurate estimations (scenario II) which was not needed in the case of $k^{*}$.

In comparison with a rigid probe (Fig. 8-e \& f), the estimated stiffness values $k^{*}$ are $\approx 10$ times smaller with MSTD values. The estimated profile is smoother with $\approx 7$ times larger MSTD value and $\approx 0.7[\mathrm{~mm}]$ different in the mean profile height between the results. The MSTD values are $\approx 10$ smaller relative to the mean measured values in the case of a rigid probe. This shows that an exact estimation is not possible due to the large MSTD in the tissue profile and stiffness estimations using a continuum probe.

Finally, Fig. 9 shows 3D maps of the mean values for the surface profile and stiffness in the probing direction $\left(k_{\mathrm{y}}\right.$ a) and indention direction $\left(k_{\mathrm{z}}-\mathrm{b}\right)$ in comparison with the estimations with a rigid probe $\left(k_{\mathrm{y} \mid \mathrm{zR}}-\mathrm{c}\right)$. The maximum value for $k_{\mathrm{y}}(\approx 50 \%$ increase for the continuum probe and $\approx 100 \%$ increase for the rigid probe w.r.t. the softer regions) occurs just before the stiff region (shown by dash line) providing predictive information about the nodule location. This value reaches its minimum almost on top of the nodule, due the small hump on top of the nodule and the fact that the nodule slightly slides back at this point. This suggests that the stiffness measurement along the probing direction ( $y$-axis) provides a rich information signal for nodule detection tasks. The maximum value for $k_{\mathrm{z}}(\approx 17 \%$ increase for the continuum probe and $\approx 100 \%$ increase for the rigid probe w.r.t. the softer regions) occurs almost on top of the nodule, providing definite information about the nodule location. The estimated value based on the base force readings is higher than the values based on direct tip force measurements $(3.4[\mathrm{~N} / \mathrm{m}](10 \%)$ for $k_{\mathrm{y}}$ and $15[\mathrm{~N} / \mathrm{m}](16 \%)$ for $k_{\mathrm{y}}$ ), showing the advantage of having direct tip force readings for better surface stiffness estimation. The estimated stiffness values and surface profile irregularities are larger in the case of a rigid probe showing the advantage of the stiff structure of the probe for more accurate and repeatable measurements. Smaller variations in measuring $k_{\mathrm{y}}$ suggests that the stiffness estimation in the probing direction is more reliable for hard nodule detection in a soft tissue sample [28].

Compared to the similar efforts in the literature, the presented methods, does not need a complex controller design [7], special considerations for guaranteeing convergence and accuracy of BVP numerical solvers [26], or suffer accuracy issues due to discretization consideration [15]. The appendage tip remains unoccupied with any force sensor meaning easier device sterilization and even the possibility of using a singleuse probe, using tip space for placement of any other tool. Finally, the fact that the presented shape and force estimation methods do not rely on any shape sensors [10], means they can be employed effectively as good basis models for Nonlinear Kalman Filter design if position measurements are not easily possible [2]. However, the large difference between the estimated values by the continuum and rigid probe suggest the importance of employing a stiffness controllable structure as in [29] to improve the estimation accuracy. Furthermore, we plan to investigate the effective palpation velocity and induced force, and comparing the results with the employed techniques by physicians, as in [28]. We consider removing the phantom slider and probing a tissue by controlling the appendage stiffness, motion, and contact force.

\section{CONCLUSION}

In this paper, a continuum appendage shape and tip loads are estimated based on real-time forward integration of Cosserat rod theory that solely relies on load readings from a base-fixed 6-axis force sensor. For the first time, this approach is employed to use a 3-DOF hydraulic-actuated braided continuum appendage for stiffness probing of a soft tissue. This method benefits from a robust light-weight easy-to-sterilize design featuring safe interaction due to the inherent compliance of the appendage soft structure. This removes the need for bulky and complex position tracking sensors or a tip force sensor. Multiple probing runs with different actuation pressures are 

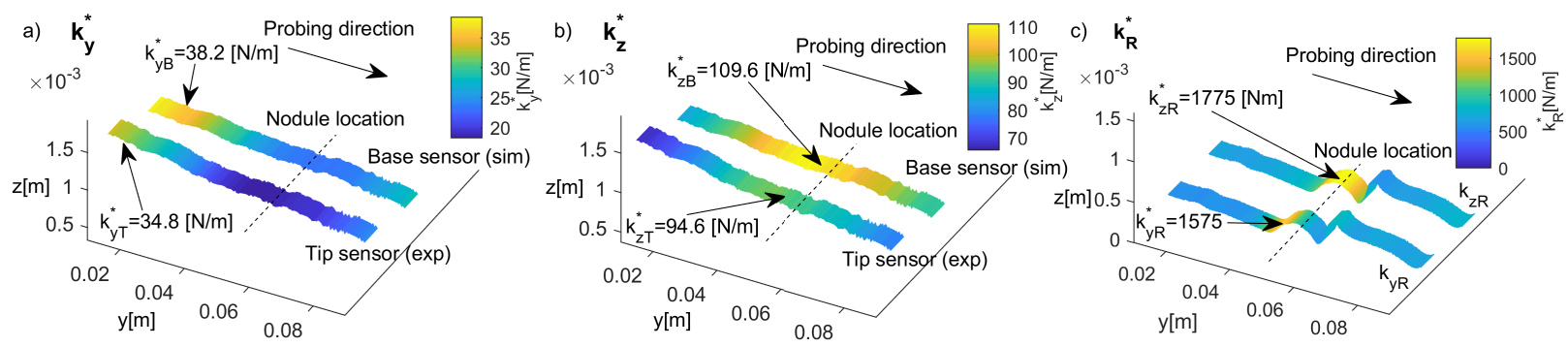

Fig. 9: The 3D surface profile and stiffness color map. a,b) The estimated values based on tip (T) and base (B) force sensor values of the continuum appendage for the same probing path (same $x$ values). c) Estimated values based on experiments with a rigid probe.

used for mapping the tissue surface shape and directional linear stiffness along the probing direction and perpendicular to the tissue surface. The results show that it is possible to detect non-homogeneous stiffness regions, i.e. a hard nodule embedded in the soft silicon phantom, in real-time. Depending on the appendage actuation scenario (passive, bent against, and bent toward probing direction), the appendage tip force is estimated with $0.016-0.037 \mathrm{~N}$ (7-20\%) mean error in the probing and $0.02-0.1 \mathrm{~N}(6-12 \%)$ in the indentation direction, 0.17 $\mathrm{mm}(14 \%)$ mean error is achieved in estimating the surface profile, and 3.4-15 [N/m] (10-16\%) mean error is observed in evaluating tissue directional stiffness in comparison to when using a tip force sensor. We suggest that an appendage which is initially bent against the slider motion (toward the probing direction) provides better horizontal force/stiffness estimation while an opposite bend results in a better estimation in the perpendicular direction. A comparison with a rigid probe shows the importance of the structure high stiffness in precise estimations suggesting the need for a continuum appendage design with stiffness controllable structure.

\section{REFERENCES}

[1] J. Burgner-Kahrs, D. C. Rucker, and H. Choset, "Continuum Robots for Medical Applications: A Survey," IEEE Trans. on Robotics, vol. 31, pp. 1261-1280, Dec. 2015.

[2] C. Shi, X. Luo, et al., "Shape Sensing Techniques for Continuum Robots in Minimally Invasive Surgery: A Survey," IEEE Trans. on Biomedical Engineering, vol. 64, pp. 1665-1678, Aug. 2017.

[3] J. Fras, J. Czarnowski, et al., "New STIFF-FLOP module construction idea for improved actuation and sensing," in 2015 IEEE Intl. Conf. on Robotics and Automation, pp. 2901-2906, May 2015.

[4] S. Sadati, S. E. Naghibi, et al., "Mechanics of Continuum Manipulators, a Comparative Study of Five Methods with Experiments," in Towards Autonomous Robotic Systems, vol. 10454, (Surrey, UK), pp. 686-702, Springer Intl. Publishing, 2017.

[5] S. Sadati, A. Shiva, et al., "Reduced Order vs. Discretized Lumped System Models with Absolute and Relative States for Continuum Manipulators," in Robotics: Science and Systems, (Freiburg, Germany), p. 10, 2019.

[6] C. Della Santina, R. K. Katzschmann, et al., "Dynamic control of soft robots interacting with the environment," in 2018 IEEE Intl. Conf. on Soft Robotics, (Livorno, Italy), pp. 46-53, IEEE, Apr. 2018.

[7] A. Bajo and N. Simaan, "Hybrid motion/force control of multibackbone continuum robots," The Intl. J of Robotics Research, p. 0278364915584806 , July 2015.

[8] R. E. Goldman, A. Bajo, and N. Simaan, "Compliant Motion Control for Multisegment Continuum Robots With Actuation Force Sensing," IEEE Trans. on Robotics, vol. 30, pp. 890-902, Aug. 2014.

[9] L. Lindenroth, C. Duriez, et al., "Intrinsic force sensing capabilities in compliant robots comprising hydraulic actuation," in 2017 IEEE/RSJ Intl. Conf. on Intelligent Robots and Systems, pp. 2923-2928, Sept. 2017.

[10] J. Back, L. Lindenroth, et al., "New kinematic multi-section model for catheter contact force estimation and steering," in 2016 IEEE/RSJ Intl. Conf. on Intelligent Robots and Systems, pp. 2122-2127, Oct. 2016.
[11] V. Aloi and C. Rucker, "Estimating Loads Along Elastic Rods," in IEEE Intl. Conf. on Robotics and Automation, (Montreal, Canada), p. 7, IEEE, 2019.

[12] J. Barbi, Real-time Reduced Large-Deformation Models and Distributed Contact for Computer Graphics and Haptics. $\mathrm{PhD}$ thesis, Carnegie Mellon University, Pittsburgh, PA 15213, 2007.

[13] T. Bretl and Z. McCarthy, "Quasi-static manipulation of a Kirchhoff elastic rod based on a geometric analysis of equilibrium configurations," The Intl. J of Robotics Research, vol. 33, pp. 48-68, Jan. 2014.

[14] D. K. Pai, "STRANDS: Interactive Simulation of Thin Solids using Cosserat Models," Computer Graphics Forum, vol. 21, no. 3, pp. 347$352,2002$.

[15] R. Takano, H. Mochiyama, and N. Takesue, "Real-time shape estimation of Kirchhoff elastic rod based on force/torque sensor," in 2017 IEEE Intl. Conf. on Robotics and Automation, (Singapore, Singapore), pp. 25082515, IEEE, May 2017.

[16] C. B. Black, J. Till, and D. C. Rucker, "Parallel Continuum Robots: Modeling, Analysis, and Actuation-Based Force Sensing," IEEE Trans. on Robotics, vol. 34, pp. 29-47, Feb. 2018.

[17] J. Konstantinova, A. Jiang, et al., "Implementation of Tactile Sensing for Palpation in Robot-Assisted Minimally Invasive Surgery: A Review," IEEE Sensors J, vol. 14, pp. 2490-2501, Aug. 2014.

[18] R. E. Goldman, A. Bajo, and N. Simaan, "Algorithms for autonomous exploration and estimation in compliant environments," Robotica, vol. 31, pp. 71-87, Jan. 2013.

[19] J. Konstantinova, G. Cotugno, et al., "Palpation force modulation strategies to identify hard regions in soft tissue organs," PLOS ONE, vol. 12, p. e0171706, Feb. 2017.

[20] S. Sanan, S. Tully, et al., "Simultaneous Compliance and Registration Estimation for Robotic Surgery," in Robotics: Science and Systems X, Robotics: Science and Systems Foundation, July 2014.

[21] S. Sadati, S. E. Naghibi, et al., "Control Space Reduction and RealTime Accurate Modeling of Continuum Manipulators Using Ritz and Ritz-Galerkin Methods," IEEE Robotics and Automation Letters, vol. 3, pp. 328-335, Jan. 2018.

[22] A. Shiva, S. H. Sadati, et al., "Elasticity Versus Hyperelasticity Considerations in Quasistatic Modeling of a Soft Finger-Like Robotic Appendage for Real-Time Position and Force Estimation," Soft Robotics, vol. 6, pp. 228-249, Jan. 2019.

[23] S. Sadati, S. E. Naghibi, et al., "TMTDyn: A Matlab package for modeling and control of hybrid rigid-continuum robots based on discretized lumped system and reduced order models," The Intl. J of Robotics Research, vol. (accepted, in press), 2019.

[24] D. Trivedi, A. Lotfi, and C. D. Rahn, "Geometrically Exact Models for Soft Robotic Manipulators," IEEE Trans. on Robotics, vol. 24, pp. 773780, Aug. 2008.

[25] B. Balachandran and E. B. Magrab, Vibrations. Australia: Cengage Learning, 2nd ed ed., 2009. OCLC: ocn181603380.

[26] C. Duriez and T. Bieze, "Soft Robot Modeling, Simulation and Control in Real-Time," in Soft Robotics: Trends, Applications and Challenges, vol. 17, pp. 103-109, Cham: Springer Intl. Publishing, 2017.

[27] N. Sornkarn, P. Dasgupta, and T. Nanayakkara, "Morphological Computation of Haptic Perception of a Controllable Stiffness Probe," PLOS ONE, vol. 11, p. e0156982, June 2016.

[28] N. Sornkarn and T. Nanayakkara, "Can a Soft Robotic Probe Use Stiffness Control Like a Human Finger to Improve Efficacy of Haptic Perception?," IEEE Trans. on Haptics, vol. 10, pp. 183-195, Apr. 2017.

[29] N. Herzig, P. Maiolino, et al., "A Variable Stiffness Robotic Probe for Soft Tissue Palpation," IEEE Robotics and Automation Letters, vol. 3, pp. 1168-1175, Apr. 2018. 